\title{
THE PENTAGON IN THE PROJECTIVE PLANE, WITH A COMMENT ON NAPIER'S RULE
}

\author{
TH. MOTZKIN
}

1. Introduction. The relations between the 6 values of the cross ratio of 4 points obtained by permuting the points are well known $(\$ 2)$. The additional relations for the cross ratios of any 4 of 5 collinear points are given in $\$ 3.5$ points (or lines) in the plane determine cross ratios with the same relations (4.1). The vertices of a pentagon are on a conic, the sides on another, the pentagon is polar to itself for a third conic. The last may be without real points; in this case the relations between the cross ratios turn out to be equivalent to the famous rule of Napier on the 5 parts of a rectangular spherical triangle, the cross ratios becoming the squares of the sines of the 5 parts. Thus the said relations are a very simple projective formulation of Napier's rule. ${ }^{1}$ These considerations form the contents of 4.2. The meets of the diagonals of a pentagon form another pentagon, projective to the first. Repeating the procedure, we obtain a bothways infinite sequence of pentagons, converging to the vertices and sides of the common polar triangle of the 3 above-mentioned conics (4.3). In $\$ 5$ the relation between the generalized cross ratios of 6 lines (or points) in the plane is determined. Finally we remark that the study of the pentagon in the projective plane is connected, in addition to that of the rectangular spherical triangle, to the study of the triangle in the metrical plane, that is, in elementary Euclidean geometry, and may be important because of its applications to the latter subject.

2. Cross ratios of 4 elements in 1 dimension. A point $a=\left(a_{0}, a_{1}\right)$ on a line is given by a binary linear form $a x=a_{0} x_{0}+a_{1} x_{1}$ vanishing at the point. Denoting by $[a, b]$ the determinant of the coefficients $a, b$ of two linear forms, the cross ratio of the pairs $a, b$ and $c, d$ is $s=[a: b, c: d]=([a, c]:[a, d]):([b, c]:[b, d])$. Hence $[c: d, a: b]=s$, $[a: b, d: c]=1: s$. By the relation of Ptolemy-Euler-Plücker $[a, b][c, d]$ $+[a, c][d, b]+[a, d][b, c]=0$, there is $[a: c, b: d]=1-s$. Hence, $[b: c, d: a]=[a: d, c: b]=t=s:(s-1), 1: s+1: t=1.1: s, 1-s$, and $t$ may be called respectively the reciprocal, complementary, and $o p$ posite cross ratios to $s$. The 6 values $s, 1: s, 1-s, t, 1: t, 1-t$ lie, if real,

Received by the editors November 2, 1944.

1 And of its dual equivalent, cf, for example, R. E. Moritz, Napier's theorem for quadrantal triangles, Congrès International des Mathématiciens, Oslo, 1936, II, pp. 170-171. 
in the 6 intervals between $-\infty,-1,0,1 / 2,1,2, \infty$, one in each interval. We shall denote the intervals between $-\infty, 0,1, \infty$ by I, II, III; II and III, I and III, I and II are interchanged respectively by passing to $1: s, 1-s, t .4$ points in cyclic order determine two opposite cross ratios. 4 points may be ordered cyclically in 3 ways; for real points there is a natural cycle, with cross ratios in III, and two interlaced cycles. ${ }^{2}$ If each point is given by its coordinates, or coordinate, $A$, the cross ratio shall be denoted by $A, B ; C, D$, for example, $[\infty, 0 ; 1, s]=s^{3}$

3. Cross ratio of 5 elements in 1 dimension. Of 5 elements, 12 cycles and 30 cross ratios may be formed. In addition to the former relations, we have 10 identities of the type $[a: b, d: e][b: c, d: e][c: a, d: e]$ $=1$. Denoting $1:[b: c, d: e]$ by $\bar{a}$, and cyclically, we obtain $\bar{a} \cdot \bar{c}=1-\bar{b}$. 5 points are projectively determined by 2 cross ratios, say $\bar{b}$ and $\bar{d}$; $\bar{a}, \cdots, \bar{e}$ are then $1-\bar{b} / 1-\bar{b} \bar{d}, \bar{b}, 1-\bar{b} \bar{d}, \bar{d}, 1-\bar{d} / 1-\bar{b} \bar{d}$. Inverting the cyclic order does not change the cross ratio quintuple. To the $o p$ posite cycle acebd belong the opposite cross ratios $1:[c: e, b: d]$ $=\bar{a}:(\bar{a}-1)$, and so on. Of the other 10 cycles, 5 , such as $e b c d a$, are bordering cycles to $a b c d e$, and 5 are bordering to $a c e b d$; the latter are also bordering or opposite to the former. The 12-graph giving the relation of bordering, having no closed 3 -chains, is not the icosahedric graph, but has 30 closed 4 -chains. To $e b c d a$ belong the cross ratios $1-\bar{e}, 1: \bar{b}, \bar{c}:(\bar{c}-1), 1: \bar{d}, 1-\bar{a}$. For real points the natural cycle has its whole cross ratio quintuple $\bar{a}, \cdots, \bar{e}$ in II, the opposite in I. The bordering cycles have quintuples with cross ratios in II, III, I, III, II, their opposites in III, I, II, I, III.

4. Cross ratios of 5 elements in 2 dimensions. 4.1. A line $a=\left(a_{0}, a_{1}, a_{2}\right)$ in the plane is given by its equation, that is, by a ternary linear form $a x=a_{0} x_{0}+a_{1} x_{1}+a_{2} x_{2}$ vanishing at its points. If we denote by $[a, b, c]$ the determinant of the coefficients $a, b, c$ of 3 linear forms, and by $[a, b]$ the meet of $a$ and $b$, given by the coefficients of $x=\left(x_{0}, x_{1}, x_{2}\right)$ in $[x, a, b]$, the cross ratio of the meets $[a, b],[a, c]$, $[a, d],[a, e]$ equals $[a, b: c, d: e]=([a, b, d]:[a, b, e]):([a, c, d]$

${ }^{2}$ The same holds for complex points, the corresponding regions being bounded by circular arcs through $1 / 2 \pm(-3)^{1 / 2} / 2$. For example, the natural cyclic order (of $\infty, 0,1, s)$ is characterized by $|s|>1,|s|>|s-1|$.

${ }^{3}$ The fractional notation $a / b$ would lead to the typographically less convenient symbol

$$
\left[\begin{array}{ll}
A & C \\
B & D
\end{array}\right],
$$

which reflects the group properties of the cross ratio and has been proposed about 50 years ago. 
$:[a, c, e])$, as is immediately verified by assuming $a=(1,0,0)$. In addition to the relations of $\S 1$, we have the Menelaos-Möbius identity $[a, b: c, d: e][b, c: a, d: e][c, a: b, d: e]=1$, with the same consequences as in $\S 2$. This is also clear by considering the lines as elements on their common conic, say $\left(1, u, u^{2}\right)$, the cross ratio of 4 lines on the fifth being equal to that determined on any fixed tangent $(1,0,0)$, that is, to that of the meets $(0, u,-1){ }^{4}$

4.2. A pentagon consists of 5 sides $a, b, c, d, e$ and 5 vertices $[a, b]$, $[b, c],[c, d],[d, e],[e, a]$ in cyclic order. Let $C_{0}$ be the mean conic, that is, the one for which $a$ and $c$ are conjugate, and so on cyclically; then the polarity at $C_{0}$ interchanges every side $a$ and its opposite vertex $[c, d]$, and also the conics $C_{1}$ and $C_{-1}$ on which the sides and the vertices are. Let $A$ and $A^{\prime}$ be the meets of $C_{0}$ and $a$, and $\left[A, A^{\prime} ;[e, a],[a, b]\right]=a^{\prime}$. Give to $A, A^{\prime}$, and $[e, a]$ the inhomogeneous coordinates $\infty, 0$, and 1 , then the coordinates of $[a, b],[a, c]$, $[a, d]$ will be $a^{\prime},-1,-a^{\prime}$, so $\bar{a}=1:[a, b: c, d: e]=\left[a^{\prime},-1 ; 1,-a^{\prime}\right]$ $=-\left(a^{\prime}-1\right)^{2}: 4 a^{\prime}$. For $\bar{a}$ in I or III, $a^{\prime}$ is real; for $\bar{a}$ in II, $a^{\prime}$ is purely imaginary. Hence $C_{0}$ has real points except for natural cycles. For the latter, throw one of the lines to infinity; an ellipse round the opposite vertex would cut its sides, so that $C_{0}$ cannot have real points. If the pentagon is on the plane at infinity of 3 -space, $C_{0}$ may be the isotropic conic; then the corresponding spherical pentagon will have quadrantal diagonals, that is of $\operatorname{arc} \pi: 2$. The sides, or diagonal angles, are given by $[i,-i ; 0, \tan \alpha]=a^{\prime}$, whence $(i-\tan \alpha)$ $:(i+\tan \alpha)=a^{\prime}, \bar{a}=\tan ^{2} \alpha:\left(1+\tan ^{2} \alpha\right)=\sin ^{2} \alpha$; they are also the parts of any of the rectangular spherical triangles $a b c, b c d$, and so on (for example, $a b c$ has the sides $\pi: 2-\alpha, \beta, \pi: 2-\gamma$ and the angles $\delta, \epsilon$ ), the Napierian cycle becoming $\alpha \gamma \epsilon \beta \delta$. From $\bar{a} \bar{c}=1-\bar{b}$ we obtain $\sin \alpha \sin \gamma=\cos \beta$ (the sign, for the usual orientation of angles, being verified for small angles). The opposite of $\sin ^{2}$ being $-\tan ^{2}$, we have $\cot \alpha \cot \beta=\cos \delta$, the second equation of Napier. ${ }^{5}$

4.3. To the opposite line cycle acebd of a pentagon belongs the same $C_{1}$, but other vertices on a conic $C_{3}$, and a new mean conic $C_{2}$. The opposite vertex cycle on $C_{3}$ leads to $C_{5}$ and $C_{4}$, and so on. We obtain

${ }^{4}$ The relations between the cross ratios of $n+3$ elements in $n>2$ dimensions obtained by joining any 4 of them to the linear variety of the others, that is, between their mutual projective coordinates, can also be derived from identities of the types Menelaos and Ptolemy, and are therefore the same as for $n+3$ elements in 1 dimension. This can, as above, be otherwise obtained, together with the natural cyclic order, by use of the norm curve through the elements.

5 Taking the mean conic as the conic of the correspondence of Hesse, we see that the cross ratio quintuples are also connected with the mutual cross ratios of 5 pairs of points on a line, which are harmonic to each other in cyclic order. 
a bothways infinite sequence of cycles, determined by any of its cycles, and conics $C_{m}$ for every integer $m$. As the sides and vertices of a pentagon have equal cross ratios, the same cross ratios belong to all cycles of the sequence; consecutive pentagons have opposite cross ratios, and every pentagon is projective to the next but one. The projectivity $P$ between both pentagons transforms every line cycle or point cycle into the next, and $C_{m}$ into $C_{m+4}$. The polarity at $C_{0}$ inverts the whole sequence and $P$; the fixed triangle $\bar{P}$ of $P$ is, therefore, a polar triangle of $C_{0}$ (and of every $C_{2 m}$ ). We may choose $\bar{P}$ as fundamental triangle and $C_{0}$ as $x x=0$; then $a d=0, b d=0$, so that $d$ is proportional to $[a, b]$, and $P$ transforms $a=\left(a_{k}\right)$ to $a^{\prime}=\left(\lambda_{k} a_{k}\right)$ which connects $[b, d]$ and $[c, e]$. Hence $\left[a^{\prime}, b, d\right]=0,\left[a^{\prime}, b\right][a, b]=0$, whence by a formula of Lagrange $a^{\prime} a \cdot b^{2}-a^{\prime} b \cdot a b=0$; likewise $b^{\prime} b \cdot a^{2}-b^{\prime} a \cdot a b$ $=0$, so $a^{\prime} a \cdot b^{2}=b^{\prime} b \cdot a^{2}$. We see that $a^{\prime} a: a a=q$ is the same for $a, \cdots, e$, so that $C_{1}$, given by $a^{\prime} a-q a a=0$, and hence or similarly every $C_{2 m+1}$, has also $\bar{P}$ as polar triangle. $\lambda$ and the cross ratio quintuple determine each other. By Poncelet's closure theorem, to a sequence of conics there belong one-dimensionally many $\left(\infty^{1}\right)$ sequences of pentagons. For a sequence of pentagons consisting of natural cycles and their opposites (as in case of the quadrantal pentagon), there are mean conics without real points, so the vertices and sides of $\bar{P}$ are real; if $\left|\lambda_{0}\right|<\left|\lambda_{1}\right|<\left|\lambda_{2}\right|$, the sides of the pentagons converge for $m \rightarrow+\infty$ to $(0,0,1)$, the vertices to $(1,0,0)$, and vice versa for $m \rightarrow-\infty$. Otherwise, that is, for a sequence of cycles bordering to natural and their opposites, only one vertex $v$ and side $s$ of $\bar{P}$ are real, for $C_{-1}$ and $C_{3}$ have exactly 2 real meets, as is easily seen for particular cases and therefore generally true, a change in the reality of $\bar{P}$ being only possible for touching $C_{-1}$ and $C_{1}$, whereas touching conics have no closure polygons of Poncelet. $P$ being projective to a transformation of similitude, the vertices of the pentagons converge to $v$, while the sides approach $v$ without converging to any line through $v$; vice versa for $s$. The limit points $(1,0,0),(0,0,1), v$ are roots of cubic equations, which might lead to an approximative construction of the roots of cubic equations. Among the projectivities belonging to the 12 point cycles of a given line cycle it is easy to find pairs whose product has a line of fixed points.

5. Cross ratios of 6 elements in 2 dimensions. The generalized cross ratio $[a: b, c: d, e: f]=[a, c: d, e: f]:[b, c: d, e: f]^{6}$ can be in 3

- For general quantics cf. my papers The hypersurface cross ratio, and $A$ 5-curve theorem generalizing the theorem of Carnot, Bull. Amer. Math. Soc. vol. 51 (1945) pp. 976-984, 972-975. 
ways considered as ratio of ordinary cross ratios; hence for each of 3 given pairs of lines the ratio of the cross ratios determined on its lines by the two other pairs is the same. If the third pair (of points) is $I, J$, then $i l n / 2$ of the (generalized) cross ratio is the difference of the angles at which one pair is seen from the points of the other. The cross ratio is 1 if, and only if, the 6 points (lines) are on the same conic - in particular, if two pairs are collinear (concurrent). There are again 30 cross ratios. The multiplicative relations of $\$ \$ 2-3$ subsist and allow us to obtain the other values from $\bar{a}=[a: b, c: d, e: f]$, $\bar{b}=[b: c, d: e, a: f], \cdots, \bar{e}=[e: a, b: c, d: f]$. As 6 lines are projectively determined by 4 constants, there must be a relation between $\bar{a}, \cdots, \bar{e}$. Indeed, if $a^{\prime}=1:[f, a: b, c: d], a^{\prime \prime}=1:[e, a: b, c: d]$, and so on, then $a^{\prime}, \cdots, e^{\prime}$ and $a^{\prime \prime}, \cdots, e^{\prime \prime}$ are cross ratio quintuples as in $\S \S 3-4$, and $\bar{a}, \cdots, \bar{e}$ equal $\left(\left(1-b^{\prime}\right):\left(1-b^{\prime} d^{\prime}\right)\right):\left(\left(1-b^{\prime \prime}\right):\left(1-b^{\prime \prime} d^{\prime \prime}\right)\right)$, $b^{\prime}: b^{\prime \prime}, \quad\left(1-b^{\prime} d^{\prime}\right):\left(1-b^{\prime \prime} d^{\prime \prime}\right), \quad d^{\prime}: d^{\prime \prime}, \quad\left(\left(1-d^{\prime}\right):\left(1-b^{\prime} d^{\prime}\right)\right):\left(\left(1-d^{\prime \prime}\right)\right.$ $\left.:\left(1-b^{\prime \prime} d^{\prime \prime}\right)\right)$. By $b^{\prime}=\bar{b} b^{\prime \prime}, d^{\prime}=\bar{d} d^{\prime \prime}$ we have $1-\bar{b} \bar{d} b^{\prime \prime} d^{\prime \prime}=\bar{c}\left(1-b^{\prime \prime} d^{\prime \prime}\right)$, $b^{\prime \prime} d^{\prime \prime}=(1-\bar{c}):(b \bar{d}-\bar{c})$. By $\left(1-\bar{b} b^{\prime \prime}\right):\left(1-b^{\prime \prime}\right)=\bar{a} \bar{c}, b^{\prime \prime}=(1-\bar{a} \bar{c}):(\bar{b}-\bar{a} \bar{c})$, and similarly $d^{\prime \prime}=(1-\bar{c} \bar{e}):(\bar{d}-\bar{c} \bar{e})$. Multiplying and equating, we get $(1-\bar{a} \bar{c})(1-\bar{c} \bar{e})(\bar{c}-\bar{b} \bar{d})=(\bar{c}-1)(\bar{b}-\bar{a} \bar{c})(\bar{d}-\bar{c} \bar{e}) . \bar{c}$ divides the difference of both sides, so finally $\bar{a} \bar{b} \bar{c} \bar{d} \bar{e}-\bar{a} \bar{c} \bar{c} \bar{e}-\bar{c} \bar{e} \bar{b}-\bar{e} \bar{b} \bar{d}-\bar{b} \bar{d} \bar{a}-\bar{d} \bar{a} \bar{c}+\bar{a} \bar{c}+\bar{c} \bar{e}$ $+\bar{e} \bar{b}+\bar{b} \bar{d}+\bar{d} \bar{a}-1=0$, an identity between the determinants of a 6, 3-matrix. Conversely, to any $\vec{a}, \cdots, \bar{e}=0,1$ with $\bar{b}=\bar{a} \bar{c}$, and so on, and fulfilling the last equation there belong certain $b^{\prime}, d^{\prime}, b^{\prime \prime}, d^{\prime \prime}$, and 6 lines not on a conic, no 3 of which are concurrent.

JERUSALEM UNIVERSITY 